\title{
Hypothesis
}

\section{Treatment of COVID-19 with Individualized Immune Boosting Interventions ${ }^{\dagger}$}

\author{
Mohan Doss, Diagnostic Imaging, Fox Chase Cancer Center, 333 Cottman Avenue, Philadelphia, PA. \\ E-mail: $\underline{\text { mohan.doss } @ \text { fccc.edu }}$
}

\begin{abstract}
There are major concerns regarding the current global pandemic of coronavirus disease 2019 (COVID-19) caused by the coronavirus SARS-CoV-2. Whereas most of the infected individuals have no symptoms or only mild symptoms, in a small minority, the infection can lead to pneumonia and death. One major concern regarding the pandemic is the $2.3 \%$ average case fatality rate of the COVID-19 patients, with the fatality rate increasing to $8 \%$ for patients aged 70 to 79 and $14.8 \%$ for those aged 80 and over. Another major concern is the person-to-person transmission of the coronavirus by asymptomatic individuals during the incubation period, making it difficult to limit the spread of the disease. Many steps are underway worldwide to limit the spread of the disease, e.g. cancellation of conferences and large gatherings, closure of schools, curtailment of travel, etc. Considering the magnitude of the adverse impact on the social, cultural, commercial, educational, scientific, health, and other aspects of the society from such steps and the large anticipated casualties with the expected spread of the disease worldwide, it is important to explore methods of reducing the fatality rates of COVID-19 patients. Since the weakness of the immune system is one of the major contributing factors for the occurrence of pneumonia, and inflammation contributes to increased mortality rates of pneumonia patients, interventions that improve the immune response and/or reduce inflammation may reduce the pneumonia incidence and mortality in COVID-19 patients. There are indeed a large number of interventions that improve the immune response and/or reduce inflammation. However, all the interventions would not be applicable or acceptable to everyone and so the interventions would need to be individualized based on individual circumstances and preferences. This approach, known as "Individualized Interventions to Improve the Immune Response", or the $\mathrm{I}^{4} \mathrm{R}$ approach needs to be tested in pilot clinical trials for the treatment of COVID-19 patients. If the pilot clinical trials demonstrate that it is effective in reducing the incidence and mortality due to pneumonia, widespread adoption of the $I^{4} \mathrm{R}$ approach for treating COVID-19 patients may reduce their morbidity and mortality, reducing the concerns regarding the coronavirus. This may be helpful in reducing the need for the drastic steps that have been taken worldwide, and help in the return of life to normalcy. Therefore, clinical trials of the $I^{4} R$ approach should be conducted on an urgent basis.
\end{abstract}

Keywords: COVID-19, coronavirus, pneumonia, I4R approach, immune system, inflammation, immune boosting interventions

\section{Background}

There are major concerns regarding the current global pandemic of coronavirus disease 2019 (COVID-19) caused by the coronavirus SARS-CoV-2.[1] Whereas most of the infected individuals have no symptoms or only mild symptoms, in a small minority the infection can lead to pneumonia and death. One major concern regarding the pandemic is the $2.3 \%$ average case fatality rate of the COVID-19 patients, with the rate increasing to $8 \%$ for patients aged 70 to 79 and $14.8 \%$ for those aged 80 and

\footnotetext{
${ }^{\dagger}$ Preprint available on OSF Preprints at: https://osf.io/6zy9u/. 
over.[2] Another major concern is the person-to-person transmission of the coronavirus by asymptomatic individuals during the incubation period,[3] making it difficult to limit the spread of the disease. The number of daily new cases in China is currently in decline following the drastic steps that were taken but the number of cases and fatalities are increasing exponentially in the rest of the world.[4] Many steps are underway worldwide to limit the spread of the disease, e.g. cancellation of conferences and large gatherings, closure of schools, curtailment of travel, etc. Considering the magnitude of the adverse impact on the social, cultural, commercial, educational, scientific, health, and other aspects of the society from such steps and the large anticipated casualties with the expected spread of the disease worldwide, it is important to explore additional methods of reducing the fatality rates of COVID-19 patients. In this article, I am proposing that COVID-19 patients be treated with exposure to low-level ionizing radiation, statins, aspirin, and other immune system boosting and/or anti-inflammatory interventions in order to reduce the occurrence of pneumonia and reduce the mortality rates of those afflicted with pneumonia.

\section{Reducing the Pneumonia Fatality Rates with Immune System Boosting and/or Anti-Inflammatory Interventions}

The weakening of the immune system is one of the major reasons for the increased incidence of pneumonia in the elderly[5] and inflammation has been implicated for the increased mortality rates in pneumonia patients.[6] Hence, an improvement in the immune system response may be effective in reducing the incidence of pneumonia and reduction of inflammation may be effective in reducing the mortality rates due to pneumonia. There are indeed a large variety of interventions that can improve the immune system response and/or reduce the inflammation, e.g. physical activity or exercise, dance, sauna, cold shower, forest bathing trips, living at a high elevation, sunlight exposure, yoga, rhythmic breathing, meditation, laughter, tai chi, acupuncture, smoking cessation, calorie restriction, intermittent fasting, fruit-vegetable consumption, vitamin D supplementation, yogurt, probiotic foods, whole grains, consumption of deuterium-depleted water, aspirin, statins, avoiding excessive alcohol consumption, reducing red meat in diet, massage, having adequate sleep, reducing light at night, reducing circadian disruption, psychological counselling, pleasurable experiences, exclusive breastfeeding, influenza and other vaccinations, exposure to low-level radiation, short-term exposure to radiofrequency radiation, radon spa therapy, ultraviolet blood irradiation, ozone therapy, hyperthermia, whole body vibration exercise, hyperbaric oxygen therapy, mixed bacteria vaccine, and infection.[7] However, all the interventions would not be applicable or acceptable to everyone and so the interventions would need to be individualized based on individual circumstances and preferences. This approach, known as "Individualized Interventions to Improve the Immune Response", or the $\mathrm{I}^{4} \mathrm{R}$ approach, has been proposed recently for preventing and treating cancer, based on the important role weakening of the immune system and chronic inflammation play in the development of clinical cancer.[7] Since the weakness of the immune system is one of the major contributing factors for the occurrence of pneumonia, and inflammation contributes to increased mortality rates of pneumonia patients, I am proposing the use of the $\mathrm{I}^{4} \mathrm{R}$ approach for treating COVID-19 patients also.

\section{Impact of Multiple Immune System Boosting and/or Anti-Inflammatory Interventions on Pneumonia Incidence and Mortality}

Let us consider the effect of three of the interventions - sauna use, statins, and acupuncture - on pneumonia incidence. Sauna use four or more times per week was associated with a reduced risk of pneumonia, with the relative risk (RR) for incidence being 0.63 (95\% CI: 0.39-1.00). [8] Those using statins had a reduced occurrence of pneumonia, with RR being 0.84 (95\% CI: 0.74-0.95).[9] Acupuncture reduced the risk of pneumonia in stroke patients with RR being 0.86 (95\% CI: 0.82- 
0.90).[10] Based on these observations, if all the three interventions are applicable and are utilized by some individuals, and we assume that the observed reductions in the pneumonia incidence due to the individual interventions are not correlated, RR for pneumonia incidence would be the product of the individual RRs, and is calculated to be 0.46 (95\% CI: $0.23,0.68)$. Though this is a rough estimate due to the simplifying assumptions made and the limited evidence available on the interventions, the $54 \%$ reduction in the pneumonia incidence from the use of these three interventions is indicative of the immense power of this approach.

Let us now consider the use of three of the interventions - low-level radiation, statins, and aspirin - for the treatment of pneumonia. Many studies have reported treating pneumonia patients by giving 30 cGy or higher radiation dose to the lungs using X-rays, and the average cure rate for all the studies was $83 \%$.[11] In one of the studies cited, X-ray treatment of pneumonia improved the fatality rate from $28 \%$, observed in prior years, to in $5.7 \%,[12]$ corresponding to RR for mortality of 0.21 (95\% CI: $0.04,0.37)$. In the same study, all the pneumonia patients who had not responded to sulfonamides, and so likely had viral pneumonia, recovered following the X-ray treatment (except for those who died within 15 hours of the treatment), indicating that the X-ray treatment was effective for viral pneumonia also. The use of Simvastatin reduced the mortality of pneumonia patients with RR being 0.50 (95\% CI: $0.13,0.88)$.[13] Aspirin therapy has been observed to reduce the mortality of pneumonia patients with RR being 0.65 (95\% CI: 0.53, 0.81).[14] If these three interventions are applicable and are utilized by some pneumonia patients, RR from the use of all the three interventions would be the product of the individual RRs (assuming that the observed reductions in the mortality rates due to the interventions are not correlated), and is calculated to be 0.07 (95\% CI: 0.0,0.14). Though this is a rough estimate due to the simplifying assumptions made and the limited evidence available on the interventions, the $93 \%$ reduction in the mortality rate from the use of these three interventions is indicative of the immense power of this approach.

Many more immune system boosting and/or anti-inflammatory interventions are available, and the use of the additional interventions, selected based on individual circumstances and preferences, i.e. the $I^{4} \mathrm{R}$ approach discussed earlier, may reduce the pneumonia incidence and mortality rates even further. Thus, this appears to be a very powerful approach indeed.

\section{Importance of Prompt Clinical Trials of Multiple Immune System Boosting Interventions for COVID-19 Patients}

Since the conventional treatments of COVID-19 patients have not been sufficiently effective and the mortality rates of the patients are high, it is incumbent upon us to test promising unconventional approaches that have a chance of reducing their mortality rates. The reasoning and evidence presented above indicate that the $\mathrm{I}^{4} \mathrm{R}$ approach may be effective in reducing the pneumonia incidence and mortality rates in COVID-19 patients. Pilot clinical trials are needed to validate this approach for the patients. For the conduct of the pilot clinical trials, the radiation dose of $30 \mathrm{cGy}$ to the lungs may be applied using the standard radiographic and fluoroscopic systems (which are readily available in hospitals worldwide) with repeated and prolonged exposures, respectively.

If the $I^{4} \mathrm{R}$ approach is found to be effective for treating COVID-19 patients in the pilot clinical trials and their mortality rates are reduced by large factors, as expected, the approach can be adopted worldwide promptly, addressing one of the major concerns regarding COVID-19, the high fatality rates in COVID-19 patients. This would decrease the COVID-19 mortality rates worldwide, reducing the fear and concerns regarding the coronavirus, and enabling a return to normalcy. Hence, pilot clinical trials of the proposed approach should be conducted on an urgent basis. 


\section{Acknowledgments}

This research was funded in part through the NIH/NCI Cancer Center Support Grant P30 CA006927.

\section{Conflict of Interests}

None.

\section{Disclaimer}

The opinions expressed in this article reflect the views of the author and do not necessarily represent the views of his employer.

\section{REFERENCES}

1. Gates, B. Responding to Covid-19 - A Once-in-a-Century Pandemic? N Engl J Med, 2020. https://www.ncbi.nlm.nih.gov/pubmed/32109012

2. Wu, Z. \& McGoogan, J. M. Characteristics of and Important Lessons From the Coronavirus Disease 2019 (COVID-19) Outbreak in China: Summary of a Report of 72314 Cases From the Chinese Center for Disease Control and Prevention. JAMA, 2020. https://www.ncbi.nlm.nih.gov/pubmed/32091533

3. Yu, P., Zhu, J., Zhang, Z., Han, Y. \& Huang, L. A familial cluster of infection associated with the 2019 novel coronavirus indicating potential person-to-person transmission during the incubation period. J Infect Dis, 2020. https://www.ncbi.nlm.nih.gov/pubmed/32067043

4. WHO. Coronavirus disease (COVID-2019) situation reports, 2020. https://www.who.int/emergencies/diseases/novel-coronavirus-2019/situation-reports

5. Meyer, K. C. The role of immunity in susceptibility to respiratory infection in the aging lung. Respir Physiol 128, 23-31, 2001. https://www.ncbi.nlm.nih.gov/pubmed/11535259

6. Curbelo, J. et al. Inflammation biomarkers in blood as mortality predictors in community-acquired pneumonia admitted patients: Importance of comparison with neutrophil count percentage or neutrophil-lymphocyte ratio. PLoS One 12, e0173947, 2017. https://www.ncbi.nlm.nih.gov/pubmed/28301543

7. Doss, M. Your Custom Roadmap for Potentially Preventing and Curing Cancer. (Mohan Doss, 2019). https://www.amazon.com/kindle/dp/B081VT8GPZ

8. Kunutsor, S. K., Laukkanen, T. \& Laukkanen, J. A. Frequent sauna bathing may reduce the risk of pneumonia in middle-aged Caucasian men: The KIHD prospective cohort study. Respir Med 132, 161-163, 2017. https://www.ncbi.nlm.nih.gov/pubmed/29229091

9. Khan, A. R. et al. The role of statins in prevention and treatment of community acquired pneumonia: a systematic review and meta-analysis. PLoS One 8, e52929, 2013. https://www.ncbi.nlm.nih.gov/pubmed/23349694

10. Chang, C. C. et al. Decreased risk of pneumonia in stroke patients receiving acupuncture: A nationwide matched-pair retrospective cohort study. PLoS One 13, e0196094, 2018. https://www.ncbi.nlm.nih.gov/pubmed/29782526

11. Calabrese, E. J. \& Dhawan, G. How radiotherapy was historically used to treat pneumonia: could it be useful today? Yale J Biol Med 86, 555-570, 2013. https://www.ncbi.nlm.nih.gov/pubmed/24348219

12. Rousseau, J. P., Johnson, W. M. \& Harrell, G. T. The Value of Roentgen Therapy in Pneumonia Which Fails to Respond to the Sulfonamides. Radiology 38, 281-289, 1942. https://pubs.rsna.org/doi/abs/10.1148/38.3.281 
13. Sapey, E. et al. Simvastatin Improves Neutrophil Function and Clinical Outcomes in Pneumonia. A Pilot Randomized Controlled Clinical Trial. Am J Respir Crit Care Med 200, 1282-1293, 2019. https://www.ncbi.nlm.nih.gov/pubmed/31206313

14. Falcone, M. et al. A Hypothesis-Generating Study of the Combination of Aspirin plus Macrolides in Patients with Severe Community-Acquired Pneumonia. Antimicrob Agents Chemother 63, 2019. https://www.ncbi.nlm.nih.gov/pubmed/30509940

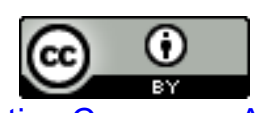

This work is licensed under a Creative Commons Attribution 4.0 International License.

Preprint available on OSF Preprints at: https://osf.io/6zy9u/ Published on March 20, 2020 